\title{
A Modular Design Approach for Ka-Band Front-End Receiver of Synthetic Aperture Interferometric Passive Imager
}

\author{
GHULAM MEHDI ${ }^{1}$, MUHAMMAD ASIF ${ }^{2}$, HU ANYONG ${ }^{2}$, JUNGANG MIAO $^{2}$ \\ ${ }^{1}$ Center for Excellence in Science \& Applied Technology (CESAT) \\ H-11/4, Islamabad \\ PAKISTAN \\ ${ }^{2}$ School of Electronics \& Information Engineering \\ Beihang University \\ 37 Xueyuan Road, Haidian District, Beijing \\ CHINA
}

\begin{abstract}
Electromagnetic Laboratory of Beihang University is engaged in the development of Ka-band synthetic aperture interferometric based passive imager named as BHU-2D-U. The imager is intended for detection of concealed weapons on human body. Noise figure of front-end receiver dictates the dynamic range and consequently the imaging performance of the imager. A modular design approach is presented to obtain improved noise figure as compared to the previous work. Additionally, the modular design gives a flexibility to adopt any desired receiver gain, instantaneous bandwidth, and noise figure and image rejection suppression. The measured gain, noise figure and image rejection is $42 \mathrm{~dB}, 4.1 \mathrm{~dB}$ and $60 \mathrm{~dB}$ respectively with $80 \mathrm{~dB}$ excellent spurious free spectral response. The reconstructed image performance of the imager is found satisfactory.
\end{abstract}

Key-Words: - millimeter-wave, multichip modules, microwave remote sensing, passive imaging

Received: June 10, 2018. Revised: October 23, 2018. Re-revised: May 16, 2019. Accepted: May 2, 2020. Published: May 29, 2020.

\section{Introduction}

With the advancement in the era of monolithic microwave integrated circuits (MMIC) technology, the focus and the thrust is shifting towards millimeter wave $(\mathrm{mmW})$ frequencies. Previously, this band has been mainly used in radio astronomy and remote sensing, but remained relatively unexplored in terms of its benefits for imaging technology, high data rate communication link, and compact radar sensors. The advancement in the device technology added with modern highprecession manufacturing technology has attracted the attention of RF system designers' to $\mathrm{mmW}$ frequency bands. The wide spectrum of applications at $\mathrm{mmW}$ frequencies encompasses from classical applications such as satellite communication, radar systems and remote sensing to emerging applications such as foreign object debris (FOD) detection radars, commercial high-data-rate wireless transfer links etc. The motivation behind this pursuance is to utilize the inherent benefits associated with $\mathrm{mmW}$ such as higher bandwidth, fine spatial resolution and producibility of miniaturized radio systems with high-level integration [1], [2]. The $\mathrm{mmW}$ system integration is primarily driven at the package-level where complex MMICs are employed. The package requirements for $\mathrm{mmW}$ are considerably different than low-frequency systems. Firstly, $\mathrm{mmW}$ packaging is sensitive to machining tolerances. Further, the effect of conducting walls on the $\mathrm{mmW}$ electromagnetic field is more pronounced than at low frequencies. In order to realize high performance $\mathrm{mmW}$ systems, there is a need of lowloss materials with well-characterized dielectric properties and added with high-resolution photolithography process technologies [1].

Before the advent of mmW MMIC devices, the $\mathrm{mmW}$ radio systems were realized with hybrid technology utilizing the discrete components[3]. A designer encountered many challenges while designing $\mathrm{mmW}$ circuits with discrete component technology. The challenges included off-chip matching of transistors, gain stabilization of amplifiers, receiver isolation etc. Further, because of the discrete nature of the design, such systems were expensive, bulky and power hungry. In system-onchip (SoC) approach, all the multi functions such as amplification, down conversion, attenuation etc. of an $\mathrm{mmW}$ radio system or sub-system is realized on a single chip. With the advancement in the device technology, such contemporary $\mathrm{mmW}$ systems are likely to be matured enough to evolve from lab 
prototypes to robust commercial products [4], [5]. The system-on-chip approach integrates various analog functions such as amplification, mixing, switching etc into a small chip. This eliminates the requirement of circuit interconnects. Other advantages are reduced mass, volume, highly repeatable electrical and low-cost fabrication. However, realization of $\mathrm{mmW}$ systems on single MMIC requires design proficiency and multiple iterations. Alternatively, MCM level design approach may be opted for the development of $\mathrm{mmW}$ radio systems [6]. One of the important dimensions of $\mathrm{mmW}$ MCM design is the manufacturing tolerances. This includes both the printed circuit tolerances as well as the mechanical tolerances.

Passive mmW imaging is a method of formation of images through detection of naturally occurring $\mathrm{mmW}$ radiation from a scene. Perhaps, the primary advantage of the passive imaging over infrared sensors is the lower attenuation in bad weather conditions such as fog, snow, clouds etc. The absorption due to fog is significantly lower in the $\mathrm{mmW}$ band as opposed to visible wavelengths. Passive imagers do not emit electromagnetic energy, thus are safe for the human body. The imager receives the spontaneous radiation emitted or reflected by the object under detection. One of the emerging applications in $\mathrm{mmW}$ bands is the passive imaging system for security screening. The concealed hazardous objects and contraband items are security threat to human safety. Such imagers have the detection capability of non-metallic contrabands such as plastic weapons concealed under clothing. The passive $\mathrm{mmW}$ imager is a good choice for such applications. Moreover, such imagers offer sufficient resolution required to detect the location and texture of the concealed objects [7], [8].

One of the passive imaging techniques is based on synthetic aperture interferometry. A synthetic aperture interferometric radiometer (SAIR) comprise of an antenna-receiver array and a crosscorrelator array. SAIR exhibits larger field of view (FOV) while maintaining the high imaging rate [9],[10]. The Microwave Laboratory of Beihang University is engaged in the development of a Kaband two-dimensional SAIR called BHU-2D-U. The BHU-2D-U imager is primarily intended for the detection of concealed weapons on human body [11]. This paper presents the design of the Ka-band front-end receiver of BHU-2D-U. A modular design methodology is adopted. The rest of the paper is arranged as follows. The section II presents a brief introduction of BHU-2D-U passive imager. Section III highlights problems of the previously reported work. The detailed design of the proposed modular front-end receiver is presented in Section IV. The design of waveguide anti-podal finline low noise amplifier (LNA), waveguide band-pass filter (BPF) and the MCM receiver are discussed. The simulation and measured results of each module are presented. The measured results of the integrated modular front-end receiver and imaging performance of BHU-2D-U are discussed in section V. Finally, the work is concluded in section VI.

\section{BHU-2D-U System Introduction}

The BHU-2D-U specifications are given in Table 1. The essence of SAIR imagery is to measure the spectral components of the brightness temperature distribution in the FOV by microwave interferometry. Each pair of antennas in the SAIR can be considered as an interferometer. With the measurement of the complex cross-correlation of the signals received by the pair of antennas, it is possible to obtain the so-called visibility function or spatial frequency [12].

Table 1. BHU-2D-U system specifications

\begin{tabular}{ll}
\hline Parameters & Specification \\
\hline Center frequency & $34 \mathrm{GHz}$ \\
Bandwidth & $400 \mathrm{MHz}$ \\
LO frequency & $32 \mathrm{GHz}($ for RF front end $)$ \\
Field of View & $22^{\circ}(\mathrm{HH}) ; 40^{\circ}(\mathrm{VV})$ \\
Synthetic Beamwidth & $1.4^{\circ}(\mathrm{HH}) ; 1.2^{\circ}(\mathrm{VV})$ \\
Temperature Sensitivity & $\sim 1.0 \mathrm{~K}$ \\
Geometry of Antenna Array & $\mathrm{U}$-shaped \\
Antenna Element Spacing & $2.62 \lambda($ horizontal $)$ \\
Receiver Type & Dual conversion \\
\hline
\end{tabular}

Each element comprises of a pyramid horn and a dual-conversion receiver with $\mathrm{I} / \mathrm{Q}$ demodulator. Coherent Los (local oscillator) are generated by a frequency synthesizer and fed into receivers through a group of dividers. Digital Signal Processing (DSP) unit computes the cross-correlations between the IF output signals of all receivers simultaneously and 
reconstruct the brightness temperature image [11]. Each receiving element in the array comprises of an element antenna and a dual-conversion receiver. The signals received by the antennas are fed to the array of dual-conversion receivers with I/Q demodulators. Each receiver in the array comprises of an $\mathrm{mmW}$ front-end and an IF module as illustrated in Fig. 1. The $\mathrm{mmW}$ front end converts the $\mathrm{mmW}$ signal into lower frequency signal, which will be then demodulated into I/Q outputs by the IF module. The nominal gain and noise figure of the receivers are $\sim 80 \mathrm{~dB}$ and $\sim 4.2 \mathrm{~dB}$ respectively. In order to equalize the gain between channels, variable attenuators are incorporated in the IF module.

\section{Previous Work}

The authors lately presented a highly integrated Ka-band receiver for the similar kind of applications [13]. It was experienced that the realization of Ka-band image rejection $\mathrm{BPF}$ and Ka-band LO harmonic suppression BPF on the laminated substrate with conventional photolithography process lacks in reproducibility of such filters in terms of electrical performance. A fabrication tolerance study has been carried out on couple of fabricated filters. In order to find out the fabrication inaccuracies, measurements were carried out on optical microscope and comparison was done with the layout pattern sent for the fabrication. It is experienced that, during the fabrication process, there is a reduction of the size from all four sides of the resonator which is typically $50 \%$ of the conductor thickness. These tolerances of the etched metal lines detune the resonators, resulting in frequency and aggravated ripples in the pass-band. Besides these, the above mentioned manufacturing issues resulted in low yield with the prescribed manufacturing process [14].

\section{Proposed Design}

The proposed modular design mitigates the manufacturing tolerance issue of the image rejection $\mathrm{BPF}$ and $\mathrm{Ka}$ band $\mathrm{LO}$ harmonic suppression BPF. The design further provides a flexibility to adopt the desired receiver gain, noise figure, instantaneous bandwidth and image rejection suppression. Brief summary and initial results were report in [15]. The proposed modular receiver comprises of three modules namely, a) antipodal finline low noise amplifier (LNA), b) waveguide band-pass filter and c) Multichip module (MCM). Some modifications have been incorporated in the modular MCM receiver. The block diagram of the modular MCM receiver is illustrated in Fig. 2. In order to attain a high yield for mass production, the IR-BPF is designed and realized in waveguide technology. It is well known from the Friis's expression (1) that the noise figure and gain of the first block in the receiver dominates the total noise figure of the system.

$$
F=F_{1}+\frac{F_{2}-1}{G_{1}}+\frac{F_{3}-1}{G_{1} G_{2}}+\ldots+\frac{F_{n}-1}{G_{1} G_{2} \cdots G_{n-1}}
$$

Where

$F=$ Total noise figure

$F_{l}=1^{\text {st }}$ stage noise figure

$G_{l}=$ Gain of $1^{\text {st }}$ stage

$n=1,2,3, \ldots \ldots \ldots \ldots$

In order to obtain improved noise figure performance, the waveguide BPF is preceded by a LNA stage. In Fig. 2, the two blocks outside the boxes are waveguide LNA and waveguide BPF modules respectively. The two boxes depict the MCM receiver. The MCM comprises of two printed circuit layers namely top and bottom layer respectively. The top layer comprises of active devices and passive structures such as filters, matching networks, operating from $2 \mathrm{GHz}$ to 34 $\mathrm{GHz}$, all realized on a single substrate for seamless integration. The blocks enclosed in the solid line box in Fig. 2 illustrate the top layer. The bottom layer comprises of chip low pass filter (LPF), IF amplifier and step-impedance BPF. The blocks enclosed in the dotted line box in Fig. 2 depict the bottom layer. Additionally, in order to avoid the requirement of Ka-band $\mathrm{LO}$ harmonic suppression BPF in the mmW LO generation block, a modified scheme is devised. The LO signal at $\mathrm{Ka}$ is generated by employing frequency multipliers. The LO multipliers chain is strategically outlined for area, power and cost efficient design.

\subsection{Antipodal LNA}

The design of the LNA being the first stage of the modular receiver is aimed for low noise figure with moderate gain. The lossy waveguide-to-printed circuit transitions deteriorate the system noise 
figure. A low-loss antipodal finline structure transition has been designed for LNA module. The antipodal finline is combined with the rectangular waveguide to transform the TE10 mode of the rectangular waveguide to the quasi-TEM mode of the microstrip line by rotating the electric field. Such kind of structure is an appropriate solution for such requirements. The antipodal finline-tomicrostrip transition has a sandwich like structure in which, as depicted in (b)

Fig. 1 (a). The rectangular Rogers RT5880 substrate $(\mathrm{Er}=2.2, \mathrm{~h}=254 \mu \mathrm{m})$ is in the center of the top and bottom copper layer. The transition is mounted across the E-plane of a waveguide. The antipodal finline is tapered from the full waveguide height to the microstrip line. Spline taper is adopted, which is demonstrated as optimal taper shape for antipodal finline transition [14]. Bond-wires are used to establish a connection between the chip and the microstrip line. However, the bond-wires introduce parasitic inductance and at MMW frequencies, such inductance value causes mismatch and results in degraded gain and noise figure. One of the methods to compensate the bond-wire inductance is to absorb such parasitic inductance into a low pass filter structure as shown in (b)

Fig. 1 (a) [15]. This T-type network is realized such as the inductor LB represents the bond-wire inductance, then, a low impedance line which acts as a shunt capacitor and finally a high-impedance actins as a series inductor. The whole cascaded structure forms a step-impedance low pass matching network These lines are much shorter than the associated guided wavelength, thus acts as quasi lumped elements. The bond-wire model and the matching network structure are designed in a fullwave EM solver. The matching network is optimized at centre frequency $(34 \mathrm{GHz})$ for low loss. The layout of the matching network along with the finline structure is shown in (b)

Fig. 1(a). The assembled LNA module has been tested on vector network analyzer (VNA). The simulated and measured results of the LNA module are in good agreement as shown in (b)

Fig. I (b). The measured LNA gain gradually reduces above $33 \mathrm{GHz}$. However, the difference between the simulated and measured gain is less than $2 \mathrm{~dB}$ at center frequency of $34 \mathrm{GHz}$. The measured noise figure of the LNA module is $3.5 \mathrm{~dB}$.

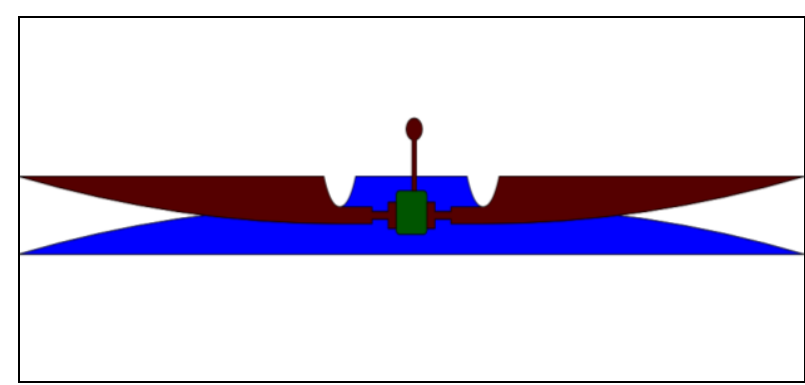

(a)

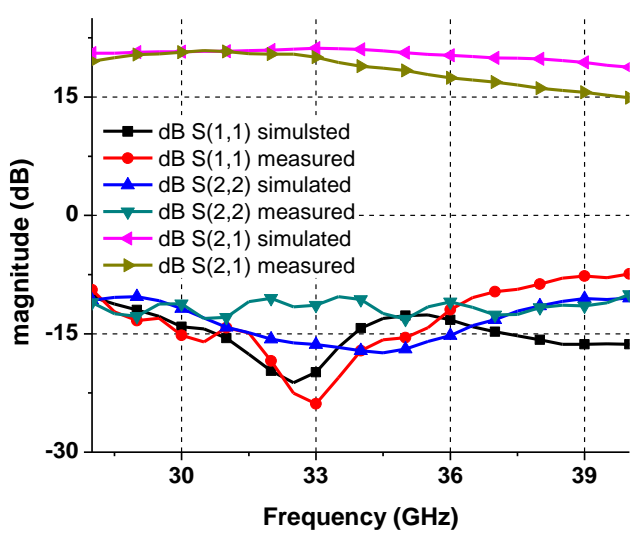

(b)

Fig. 1. (a) Antipodal finline LNA layout (b) Simulated vs. measured response of LNA module

\subsection{Waveguide Band-pass Filter}

Microstrip BPFs are compact in size and can be realized for wider operation band when compared with waveguide filter, but at the cost of larger insertion loss and poor stop band suppression performance especially at $\mathrm{mmW}$ frequencies. Waveguide filter could achieve very high Q value, low insertion loss and good temperature stability and are especially suitable for narrow-band filtering applications. In the design of direct coupling waveguide band-pass filter, commonly used inductive irises, pins, small or E insert as coupling network between levels of waveguide resonant cavity. In this work, an H-plane inductance irises structure is used to design the Ka-band rectangular waveguide BPF.

The H-plane waveguide filter uses the waveguide section of half wavelength as the series resonators uses the parallel inductance formed by inductive irises as a coupling structure between the resonators [16]. The waveguide filter is designed in HFSS for $300 \mathrm{MHz}$ bandwidth centered at $34 \mathrm{GHz}$. The chamfer factors leading to frequency offset is considered. The frequency offset can be corrected 
by adjusting the center frequency to final realizes the precise design of the filter. (b)

Fig. 2(a) depicts the fabricated BPF and the simulated and measured results of two fabricated BPFs are shown in (b)

Fig. 2(b). The measured results of two fabricated BPFs are in good agreement. The measured image rejection is better than $60 \mathrm{~dB}$.

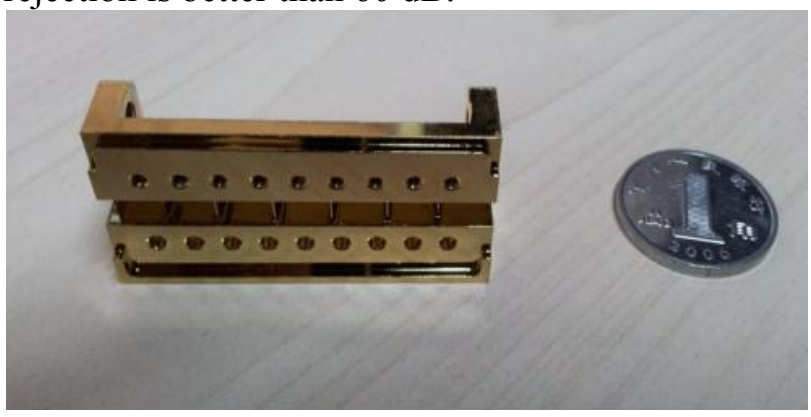

(a)

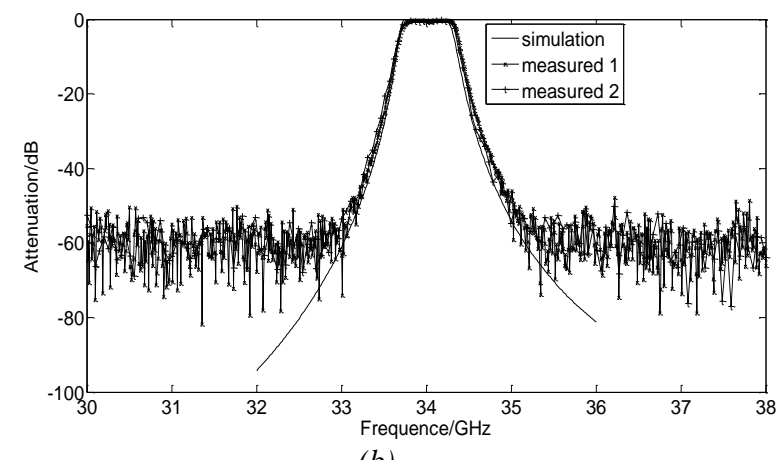

(b)

Fig. 2. (a) Waveguide BPF (b) Simulated vs. measured response of Waveguide BPFs

\subsection{Receiver}

The block diagram of the MCM receiver is illustrated in Fig.1. The complete MCM is realized on a single substrate and a two-layer planar technology is adopted for compact realization. This design scheme resulted in a low cost solution. Further, printed circuit fabrication and assembling time is significantly reduced as well. A reactively tuned waveguideto-microstrip transition is designed to interface the MCM with waveguide BPF. Oscillators are one of the key elements in $\mathrm{mmW}$ receivers. Frequency multipliers are employed for the generations of low phase noise LO signal while maintaining the high out power of $15 \mathrm{dBm}$ to drive the down conversion stage. An optimum circuit configuration in terms of space, power consumption, cost and performance is adopted to transform $4 \mathrm{GHz}$ input signal to $32 \mathrm{GHz} \mathrm{LO}$ signal. Moreover, in the strategic design of the LO chain, by selecting the appropriate multiplier chips and insertion of 8 and $16 \mathrm{GHz}$ band-pass filters, the requirement of $32 \mathrm{GHz}$ is mitigated to avoid manufacturing issues. The block diagram of the LO generation block is shown in Fig. 8. The first component in the LO block is a $2 \mathrm{GHz}$ notch filter. The $2 \mathrm{GHz}$ notch filter suppresses this half frequency component coming from the synthesizer unit by more than $50 \mathrm{~dB}$. The first active doubler converts $4 \mathrm{GHz}$ signal to $8 \mathrm{GHz}$. An X-band edge-coupled follows the active doubler. The next stage is passive doubler. A Ku-band $\mathrm{BPF}$ at $16 \mathrm{GHz}$ follows the passive doubler. The final component in the LO generation unit is an active doubler. A simulation model is developed for spectral analysis of the proposed LO generation unit. The simulated spectral response of the $\mathrm{mmW} \mathrm{LO}$ output is illustrated in (b)

Fig. 3 (a). It can been seen that the closely spaced unwanted harmonic components locating at $28 \mathrm{GHz}$ and $36 \mathrm{GHz}$ are $-95 \mathrm{dBc}$ and $-92 \mathrm{dBc}$ respectively. It is evident that the harmonic level at $8 \mathrm{GHz}$ and $16 \mathrm{GHz}$ are -55 $\mathrm{dBc}$ and $-60 \mathrm{dBc}$ which are relatively higher, however, since these harmonics lie far away from the operating bandwidth of the receiver, thus of not concern. In BHU-2D-U, the frequency synthesizer unit provides LO signals to both $\mathrm{mmW}$ MCM and IF I/Q demodulator receiver. The LO uncorrelated phase noise (PN) introduces visibility phase errors in the reconstructed image. In order to estimate the cumulative phase noise of the $\mathrm{mmW}$ LO signal due to addition of three frequency multipliers stage, a simulation model is developed. In the model, the $4 \mathrm{GHz}$ PLL synthesizer is cascaded with the mmW LO generation block. The PN at $32 \mathrm{GHz}$ at offset ranges from $1 \mathrm{~Hz}-1 \mathrm{MHz}$ is according to the theoretical expectations as illustrated in (b) 
Fig.

3

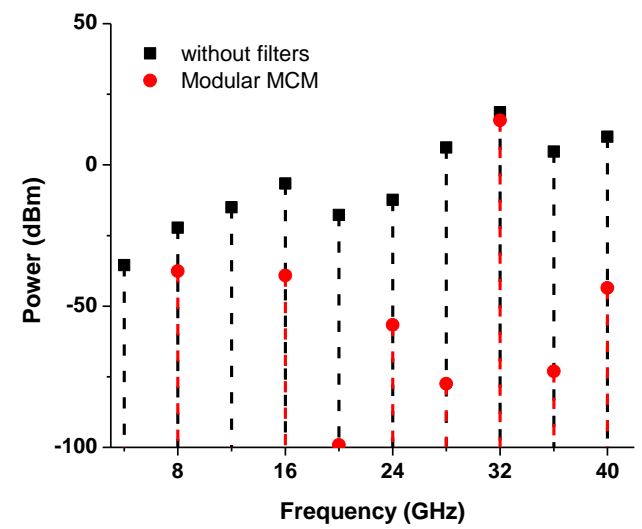

(a)

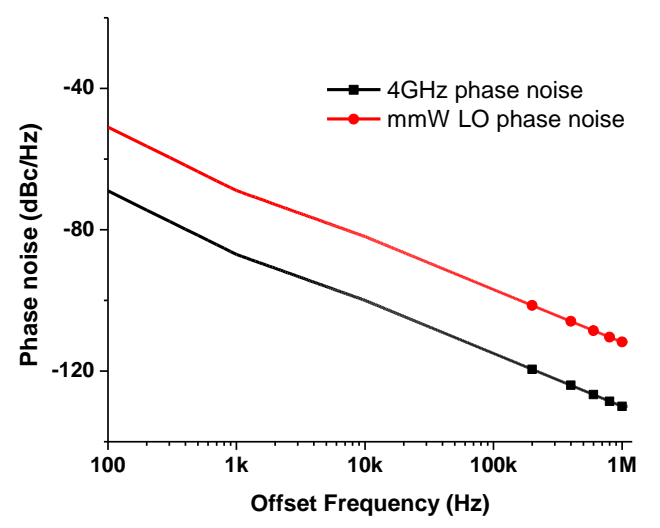

(b)

Fig. 3. (a) mmW LO spectral response with and without harmonic suppression filters (b) Simulated Lo phase noise

The MCM receiver gain and frequency response is characterized on VNA. (a)

Fig. 4 (a) shows the measured response of three MCM receivers. The measured gain is as expected above $23 \mathrm{~dB}$ in the working band of 33-35 GHz. (a)

Fig. 4 (b) depicts the photograph of the MCM receiver.

(b).

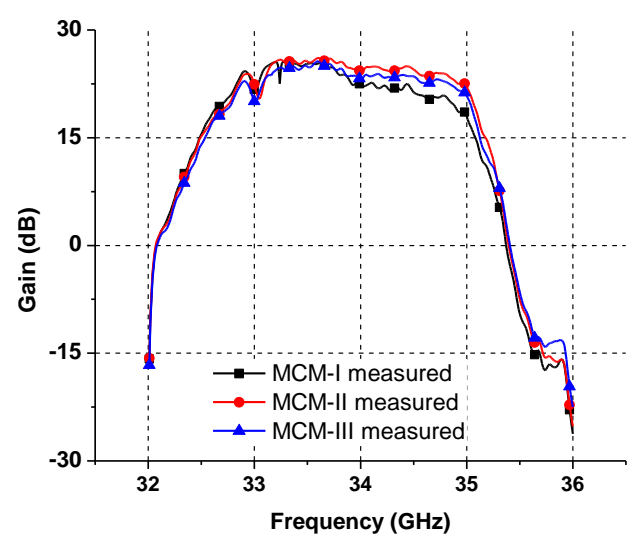

(a)

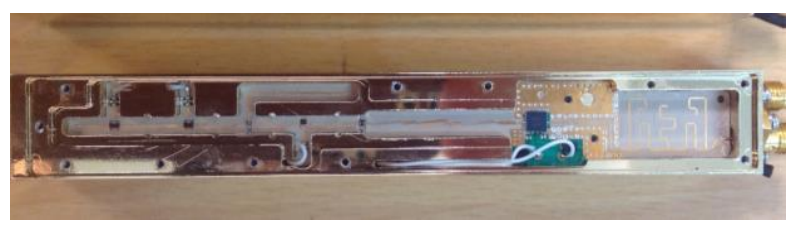

(a)

Fig. 4. (a) Measured response of three MCM receivers (b) Multi-chip module receiver (top view)

\section{Integrated Measurements and System Evaluation}

The waveguide IR-BPF follows the antipodal finline LNA stage. The IR-BPF is followed by the MCM receiver. In the MCM receiver, the die chips are eutactically attached with the metallic enclosure surface. The bond-wire lengths inside the MCM are kept as short as possible. The integrated front-end receivers are measured on VNA. The measured gain and frequency response of the three integrated receivers are shown in (b)

Fig. 5(a). The measured gain is $42 \mathrm{~dB}$ in the working band of 33.8-34.2 GHz. The image rejection is better than $60 \mathrm{~dB}$. The symmetric frequency response of the three fabricated front-end receivers indicates that the fabrication accuracy is satisfactory and is suitable for receiving array elements. In order to perform the spectrum analysis of the front-end receiver, the input of the LNA module is terminated to a matched load. The $4 \mathrm{GHz}$ LO signal is fed from the BHU-2D-U synthesizer unit. The IF output the MCM is analyzed on the spectrum analyzer. The measured output spectrum is shown in (b)

Fig. 5(b). The spectral analysis of the front-end MCM exhibits more than $80 \mathrm{~dB}$ spurious free output response. 


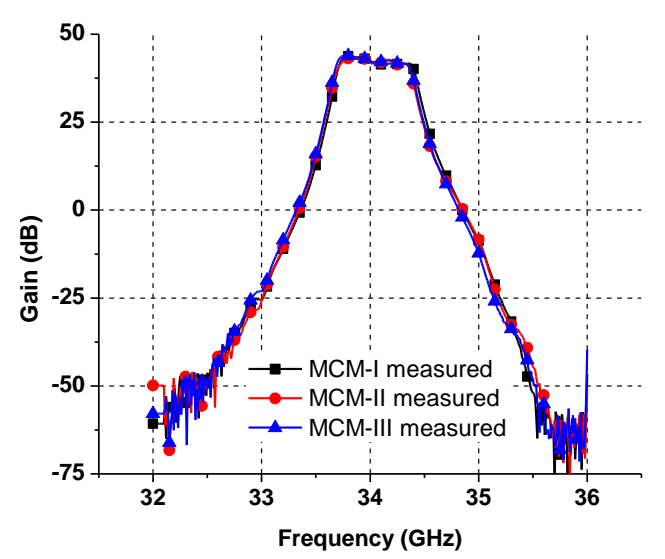

(a)

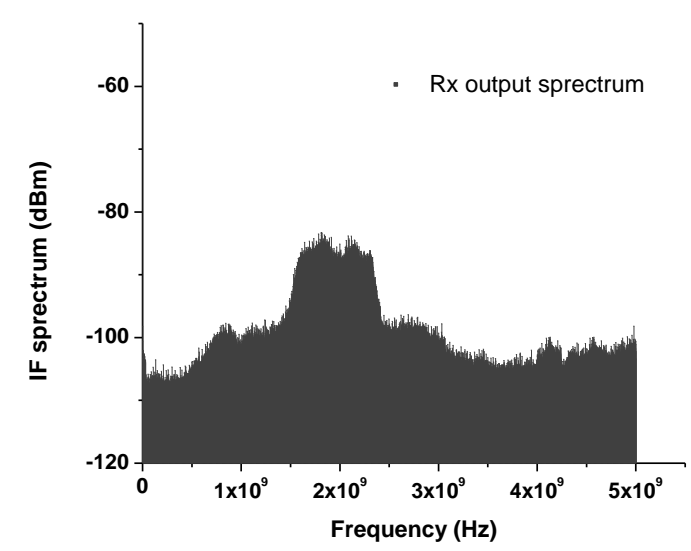

(b)

Fig. 5. Measured response of 03 integrated MCMs (b). Measured output spectrum, input terminated to matched load

BHU-2D-U system as depicted in Fig.9 (a) has been developed and presently the system is undergoing though the test and evaluation. In order to perform imaging experiment on a person, a metal sheet with $45^{\circ}$ inclined to the ground is fixed behind the person to establish a uniform background. The integration time in the experiment is set to $0.5 \mathrm{~s}$. Fig. 6 (b) shows the picture of a person holding a metallic strip under the clothing. The corresponding image reconstructed by BHU-2D-U is illustrated in Fig. 6(c). It is evident that the image constructed by BHU-2D-U is in good match with the optical image. The concealed metallic strip has been detected and can be distinguished in the $\mathrm{mmW}$ image. However, the brightness temperature distribution of the person is not uniform. One of the possible reasons is the reflection from the ambient environment. The quality of the image (concealed metallic strip) recognized and reconstructed by the $\mathrm{mmW}$ imager is satisfactory.

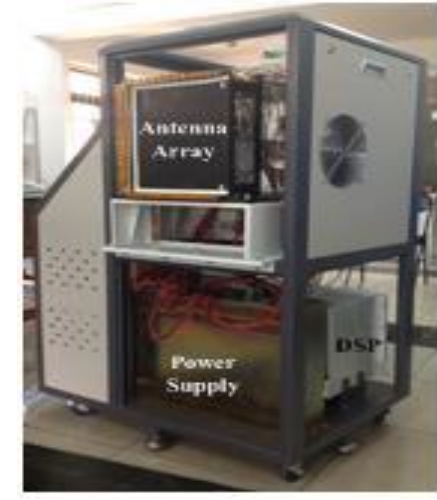

(a)

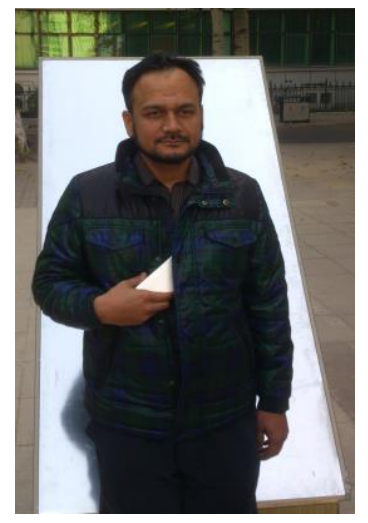

(b)

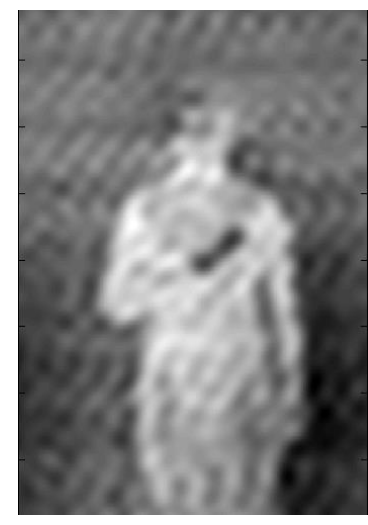

(c)
Fig. 6. (a) BHU-2D-U Passive Imager (b) Optical image of a person with concealed metallic strip (c) Corresponding $\mathrm{mmW}$ image

\section{Conclusion}

The 2-D SAIR based passive MMW imagers offer distinct advantages such as larger field of view (FOV) and high imaging rate. In this work, a modular front-end receiver is presented for a SAIR based passive imager. The measured receiver gain, noise figure and image rejection is $42 \mathrm{~dB}, 4.1 \mathrm{~dB}$ and $60 \mathrm{~dB}$ respectively with more than $80 \mathrm{~dB}$ excellent spurious free spectral response. The reproducibility of the modular front-end receiver is good with high yield. Further, the system level experiments of BHU-2D-U shows that the performance of modular front-end receiver is satisfactory and system is capable of detecting concealed objects.

\section{References:}

[1] D. Liu, U. Pfeiffer, J. Grzyb, and B. Gaucher, Advanced millimeter-wave technologies: antennas, packaging and circuits. John Wiley \& Sons, 2009.

[2] G.Mehdi and Jungang Miao, Multi-layer System-on-Substrate for Ka-band Foreign Object Debris (FOD) Detection Sensor, 
WSEAS Transactions On Circuits and Systems, E-ISSN: 2224-266X, Volume 14, 2015

[3] J. C. Wiltse, "History of millimeter and submillimeter waves," Microw. Theory Tech. IEEE Trans., vol. 32, no. 9, pp. 1118-1127, 1984.

[4] Chen, C., Mehdi, G., Wang, C., Dilshad, U., Hu, A. and Miao, J., 2019. A GaAs Power Detector Design for C-band Wideband Complex Cross-Correlation Measurement. IEEE Transactions on Instrumentation and Measurement.

[5] A. Tomkins, P. Garcia, and S. P. Voinigescu, "A passive W-band imaging receiver in $65-$ nm bulk CMOS," Solid-State Circuits, IEEE J., vol. 45, no. 10, pp. 1981-1991, 2010.

[6] M. Morgan, S. Weinreb, N. Wadefalk, and L. Samoska, "A MMIC-based 75-110 GHz signal source," in Microwave Symposium Digest, 2002 IEEE MTT-S International, 2002, vol. 3, pp. 1859-1862.

[7] Guo, X., Mehdi, G., Asif, M., Hu, A. and Miao, J., 2019. 1-Bit/2-Level Analog-toDigital Conversion Based on Comparator and FPGA for Aperture Synthesis Passive Millimeter-Wave Imager. IEEE Access, 7, pp.51933-51939.

[8] Asif, M., Guo, X., Miao, J. and Mehdi, G., 2019, January. Coarsely quantized digital correlators for passive millimeter wave imagers: A hardware perspective. In 2019 16th International Bhurban Conference on Applied Sciences and Technology (IBCAST), pp. 412-418.
[9] N. Skou, "Microwave radiometer systems: design and analysis," Norwood, MA, Artech House, 1989, 171 p., vol. 1, 1989.

[10] C. Zheng, X. Yao, A. Hu, and J. Miao, "A Passive Millimeter-Wave Imager Used for Concealed Weapon Detection," Prog. Electromagn. Res. B, vol. 46, pp. 379-397, 2013.

[11] C. Zheng, X. Yao, A. Hu, and J. Miao, "Initial Results of a Passive Millimeter-Wave Imager Used for Concealed Weapon Detection Bhu-2D-U," Prog. Electromagn. Res. C, vol. 43, pp. 151-163, 2013.

[12] I. Corbella, A. Camps, F. Torres, and J. Bará, "Analysis of noise-injection networks for interferometric-radiometer calibration," Microw. Theory Tech. IEEE Trans., vol. 48, no. 4, pp. 545-552, 2000.

[13] G. Mehdi, H. Anyong, and J. Miao, "A Highly Integrated $\mathrm{Ka}$ Band Front-End Receiver," Int. J. Comput. Sci. Issues, vol. 10, no. 3, pp. 271-279, 2013.

[14] B. Yang, G. Mehdi, A. Hu, Y. Xie, X. Yao, J. Miao, and J. Zhang, "The round-ended design and measurement of all symmetric edge-coupled bandpass filter," Prog. Electromagn. Res. C, vol. 38, pp. 191-203, 2013.

[15] G. Mehdi, Hu Anyong, Yong Fu, Yu Tongfei, Jungang Miao, and Zafar Bedar Khan. "A modular Ka-band front-end receiver for passive imaging system." In INMIC, 2013 16th International, pp. 121124. IEEE, 2013.

[16] R. M. Kurzrok, "General three-resonator filters in waveguide," Microw. Theory Tech. IEEE Trans., vol. 14, no. 1, pp. 46-47, 1966.

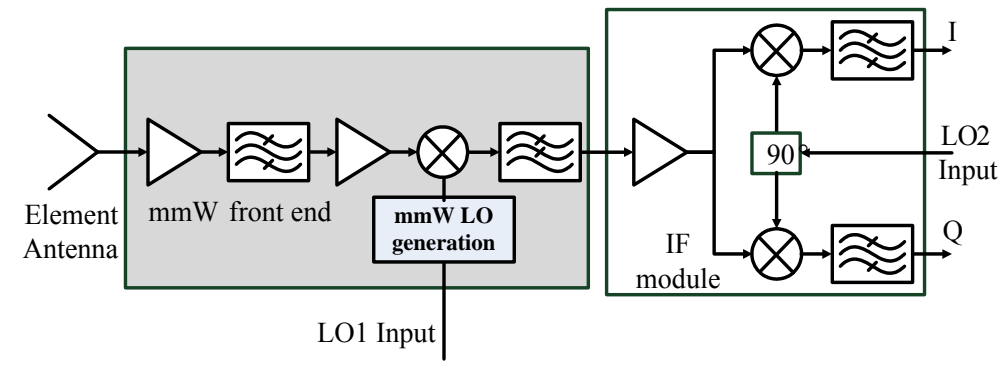

Fig. 7. System configuration of $B H U-2 D-U$ 


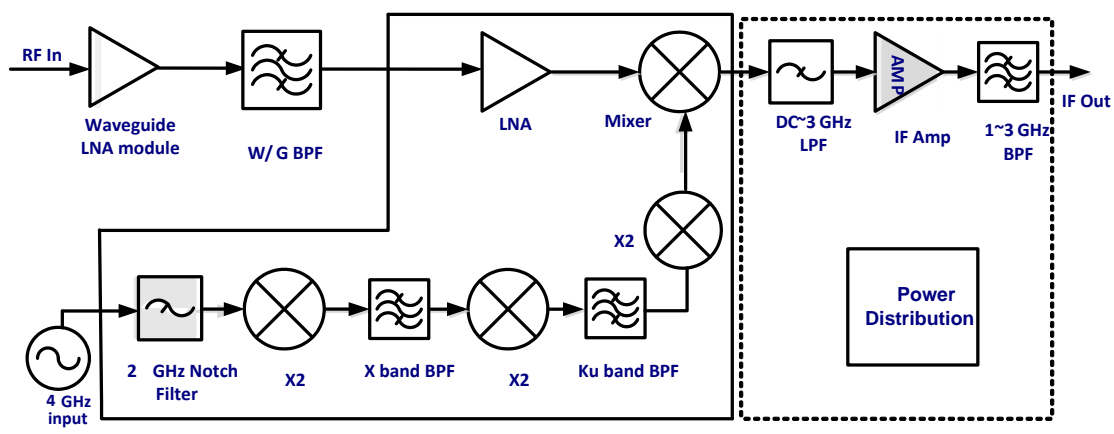

Fig. 2. Functional block diagram of $B H U-2 D-U$

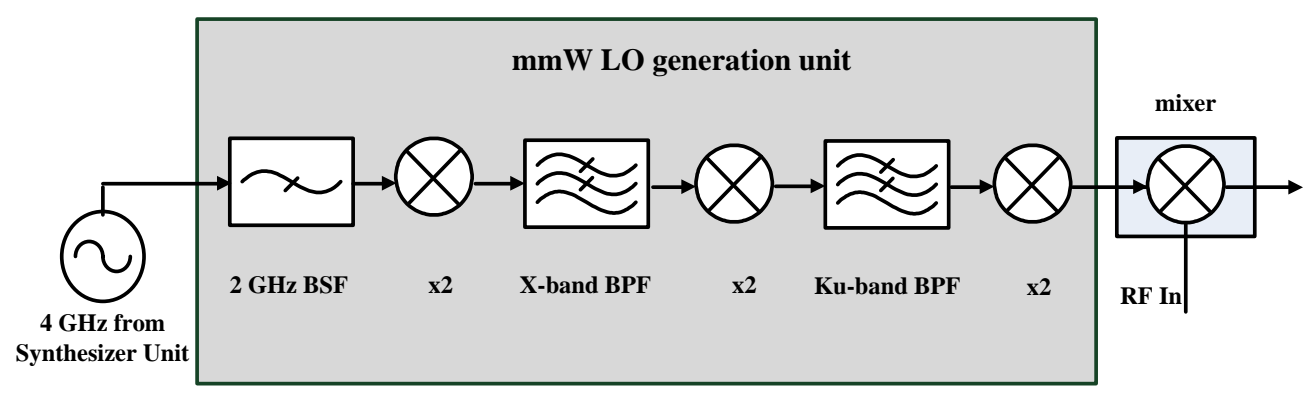

Fig. 8. Block diagram of LO generation unit 